\title{
On two-loci of metric projections
}

\author{
By
}

\author{
F. S. DE BLASI and N. V. ZhIVKOV*)
}

\begin{abstract}
A typical closed and bounded subset $M$ of a separable strictly convex Banach space $X$ generates a metric projection (nearest point mapping) which is twovalued on a dense and everywhere continual subset $C_{M}$ of $X$.
\end{abstract}

1. Introduction. Let $(X,\|\cdot\|)$ be a Banach space. A non-empty set $M \subset X$ generates a distance function $d(x, M)=\inf \{\|x-z\|: z \in M\}$ and a set-valued mapping

$$
P(x, M)=\{y \in M:\|x-y\|=d(x, M)\}
$$

called metric projection or nearest point mapping. Each $y \in P(x, M)$ is called a (point of) best approximation of $M$ from $x$.

With every metric projection mapping $P(\cdot, M)$ and every integer $n \geqq 0$ we associate the set

$$
L^{n} M=\{x \in X: \operatorname{card} P(x, M)=n\}
$$

called $n$-locus of $P$ generated by $M$ or simply $n$-locus of $M$, when there is no ambiguity what the projection mapping is (here card stands for the cardinality number of a set). For instance: $L^{0} M$ is the set of the elements of $X$ for which there is no point of best approximation in $M$ and $L^{1} M$ is the set of points admitting a unique best approximation in $M$. Denote by $A(M)$ the set of all points with at least two best approximations in $M$. This set is called ambiguous locus of $M$ and obviously $\bigcup_{n=2}^{\infty} L^{n} M \subset A(M)$.

A number of papers have been devoted to the investigation of different aspects of the sets $L^{n} M$. Our interest in the present note is in those results which involve Baire category.

From this point of view one of the first classical results was established in 1963 by Stechkin [9] who showed that for each closed and nonempty set $M$ of a uniformly rotund Banach space $X$ the set $L^{1} M$ is residual (i.e. it contains a dense $G_{\delta}$ subset of $X$ ). For developments of this line of research we mention, among others, the contributions in [7], [5], [3], [4].

The study of the ambiguous locus for typical closed bounded sets was initiated more recently by Zamfirescu [10]. Denote by $\mathscr{B}(X)$ the space of all bounded closed and nonempty subsets of $X$, endowed with the Hausdorff metric $H$. Although $L^{1} M$ is residual for every

Mathematics Subject Classification (1991): Primary 41A65; Secondary 54E52, 46B20.

*) Research of the second author is in part supported by the National Fund for Scientific Research at the Bulgarian Ministry of Science and Education under contract MM406/94, and in part by Consiglio Nazionale delle Ricerche, G.N.A.F.A., Italy. 
$M \in \mathscr{B}\left(\mathbb{R}^{n}\right)$, for a typical set $M$ in $\mathbb{R}^{n}$ (in the sense of Baire category again) the set $A(M)$ is dense in $\mathbb{R}^{n}$ [10]. This approach was developed by several authors including [1], [2], [11], [12].

A set $C$ is called everywhere continual in $X$ if $\operatorname{card}(C \cap U)$ is not smaller than the continuum, for every nonempty open $U \subset X$.

The aim of this note is to establish the following result:

Theorem. Let $X$ be a separable strictly convex Banach space with $\operatorname{dim} X \geqq 2$. Then there exists a residual subset $\mathscr{R}$ of $\mathscr{B}(X)$ such that for every $M \in \mathscr{R}$ the two-locus $L^{2} M$ contains an everywhere continual (hence dense) in $X$ subset $C_{M}$ at each point of which $P$ is upper semicontinuous.

2. More notation. Let $X$ be a Banach space, and let $\theta$ be its origin. The closed line segment with end-points $x, y \in X$ is denoted by $[x, y]$. Further we put $(x, y]=[x, y] \backslash\{x\}$, $[x, y)=[x, y] \backslash\{y\}$ and $(x, y)=[x, y] \backslash\{x, y\}$.

For a subset $M$ of $X, \operatorname{int} M, \operatorname{cl} M, \operatorname{bd} M, \operatorname{co} M, \operatorname{diam} M$ and $\operatorname{card} M$ stand for the interior, closure, boundary, convex hull, diameter and cardinality of $M$ respectively. The set usc $M$ consists of all points $x \in X$ at which $P(x, M)$ is upper semi-continuous. If $M_{i} \subset X, i=1,2$, and $\lambda$ is a real number then $M_{1}+\lambda M_{2}=\left\{x+\lambda y: x \in M_{1}, y \in M_{2}\right\}$.

$B[x, r]$ is the closed ball centered at $x \in X$ with radius $r>0$, while $B(x, r)$ (resp. $S(x, r)$ ) is the open ball (resp. the sphere) with the same center and radius.

Suppose $M_{1}$ and $M_{2}$ are non-empty closed subsets with empty intersection. Denote the equidistant hypersurface from $M_{1}$ and $M_{2}$ by

$$
\sigma\left(M_{1}, M_{2}\right)=\left\{x \in X: d\left(x, M_{1}\right)=d\left(x, M_{2}\right)\right\} .
$$

Obviously, $\sigma\left(M_{1}, M_{2}\right)$ is a closed set in $X$, hence it is a complete metric space under the metric induced by $\|\cdot\|$.

$H\left(M_{1}, M_{2}\right)$ is the Hausdorff distance between the sets $M_{1}$ and $M_{2}$. In the sequel by Hausdorff topology (of $\mathscr{B}(X)$ ) we mean the topology of $\mathscr{B}(X)$ generated by the Hausdorff distance $H$. It is well known that $(\mathscr{B}(X), H)$ is a complete metric space.

For the sake of simplicity of the notation, the balls in $\mathscr{B}(X)$ are denoted by a different letter, i.e. $\mathcal{O}(M, r)$ (resp. $\mathcal{O}[M, r])$ stands for the open (resp. closed) ball, centered at $M \in \mathscr{B}(X)$ with radius $r>0$.

A point-to-set mapping $F: Z \rightarrow Y$, where $Z$ and $Y$ are topological spaces is upper semicontinuous at $z_{0} \in Z$ provided for every open set $U, U \supset F\left(z_{0}\right)$ there is an open set $V \subset Z$, $z_{0} \in V$, such that $U \supset F(z)$ whenever $z \in V$.

As usual c denotes the cardinal number of the continuum.

3. The lemmas. This sections contains the auxiliary results we need for the proof of the Theorem. Throughout $X$ is a strictly convex Banach space $(\operatorname{dim} X \geqq 2$ ). The following lemma makes sharper (for the uniqueness part) a result proved in [1].

Lemma 1. Suppose $y_{1}$ and $y_{2}$ are two elements of $X$ such that $\left\|y_{1}\right\|=\left\|y_{2}\right\|=r, y_{1} \neq \pm y_{2}$. Suppose $t_{0} \in(0,1)$. Then there exist $\gamma>0$ and $\delta>0$, such that for every two closed and 
nonempty sets $M_{i} \subset B\left[y_{i}, \gamma\right], i=1,2$, and for every $t \in\left[t_{0}-\delta, t_{0}+\delta\right]$ there exists a unique $x(t) \in\left[t y_{1}, t y_{2}\right] \cap \sigma\left(M_{1}, M_{2}\right)$, i.e. $\{x(t)\}=\left[t y_{1}, t y_{2}\right] \cap \sigma\left(M_{1}, M_{2}\right)$.

Proof. For $t \in(0,1]$ denote by $l_{t}$ the straight line through $t y_{1}$ and $t y_{2}$, and by $l_{0}$ the line through $\theta$, parallel to $l_{1}$. Set also

$$
\left\{z_{i}\right\}=P\left(y_{i}, l_{0}\right), \quad\left\{u_{i}(t)\right\}=l_{t} \cap\left(y_{i}, z_{i}\right), \quad i=1,2, \quad t \in(0,1) .
$$

Note that $\theta \in\left(z_{1}, z_{2}\right)$, since $\left(y_{1}, y_{2}\right) \subset B(\theta, r)$ by the strict convexity of $X$, and that the unique best approximation of $l_{1}$ from $\theta$ lies in $\left(y_{1}, y_{2}\right)$. Obviously, $\left[t y_{1}, t y_{2}\right] \subset\left(u_{1}(t), u_{2}(t)\right)$ whenever $t \in(0,1)$. Now, for the sake of convenience we define the functions

$$
\phi_{i}(t, s)=\left\|y_{i}-(1-s) u_{1}(t)-s u_{2}(t)\right\|, \quad(t, s) \in(0,1) \times \mathbb{R}, \quad i=1,2 .
$$

Since the norm is strictly convex, for each $t \in(0,1)$ and $i=1,2$ the function $\phi_{i}(t, \cdot)$ is strictly convex with respect to $s$. On the other hand, $\phi_{1}(t, \cdot)$ attains its minimum at 0 and $\phi_{2}(t, \cdot)$ attains its minimum at 1 , so for every $t \in(0,1) \phi_{1}(t, \cdot)$ is strictly increasing as a function of $s \in[0,1]$ while $\phi_{2}(t, \cdot)$ is strictly decreasing on the same interval.

Let $s_{i}(t)$ be the solution of $t y_{i}=(1-s) u_{1}(t)+s u_{2}(t)$ for $i=1,2$ and $t \in(0,1)$. We have

$$
\phi_{1}\left(t_{0}, 0\right)<\phi_{1}\left(t_{0}, s_{1}\left(t_{0}\right)\right), \quad \phi_{2}\left(t_{0}, s_{2}\left(t_{0}\right)\right)>\phi_{2}\left(t_{0}, 1\right) .
$$

On the other hand, the strict convexity of the norm implies

$$
\phi_{1}\left(t_{0}, s_{1}\left(t_{0}\right)\right)<\phi_{2}\left(t_{0}, s_{1}\left(t_{0}\right)\right), \quad \phi_{1}\left(t_{0}, s_{2}\left(t_{0}\right)\right)>\phi_{2}\left(t_{0}, s_{2}\left(t_{0}\right)\right) .
$$

Now, since all functions involved in (1) and (2) are continuous, there is $\delta>0$ such that for $\Delta=\left[t_{0}-\delta, t_{0}+\delta\right]$ the following inequalities are true:

$$
\begin{aligned}
& A_{1}=\max \left\{\phi_{1}(t, 0): t \in \Delta\right\}<B_{1}=\min \left\{\phi_{1}\left(t, s_{1}(t)\right): t \in \Delta\right\} \\
& B_{2}=\min \left\{\phi_{2}\left(t, s_{2}(t)\right): t \in \Delta\right\}>A_{2}=\max \left\{\phi_{2}(t, 1): t \in \Delta\right\} \\
& C_{1}=\max \left\{\phi_{1}\left(t, s_{1}(t)\right): t \in \Delta\right\}<D_{1}=\min \left\{\phi_{2}\left(t, s_{1}(t)\right): t \in \Delta\right\} \\
& D_{2}=\min \left\{\phi_{1}\left(t, s_{2}(t)\right): t \in \Delta\right\}>C_{2}=\max \left\{\phi_{2}\left(t, s_{2}(t)\right): t \in \Delta\right\} .
\end{aligned}
$$

Choose $\gamma>0$ so that

$$
\gamma<2^{-1} \min \left\{\left\|y_{1}-y_{2}\right\|, B_{i}-A_{i}, D_{i}-C_{i}: i=1,2\right\} .
$$

Suppose $M_{1}$ is a closed nonempty subset of $B\left[y_{1}, \gamma\right]$, and fix $t \in \Delta$. We will show that the function $d_{1}(s)=d\left((1-s) u_{1}(t)+s u_{2}(t), M_{1}\right)$ is strictly increasing on the segment $\left[s_{1}(t), s_{2}(t)\right]$.

Assuming the contrary, there are $r_{1}, r_{2} \in\left[s_{1}(t), s_{2}(t)\right]$, with $r_{1}<r_{2}$, such that $d_{1}\left(r_{1}\right) \geqq d_{1}\left(r_{2}\right)$. Put $x_{i}=\left(1-r_{i}\right) u_{1}(t)+r_{i} u_{2}(t), i=1,2$. We have

$$
A_{1}+\gamma<d_{1}\left(r_{1}\right) \text {. }
$$

Indeed, let $y \in M_{1}$ be arbitrary. Since

$$
B_{1} \leqq\left\|t y_{1}-y_{1}\right\| \leqq\left\|x_{1}-y_{1}\right\| \leqq\left\|x_{1}-y\right\|+\gamma<\left\|x_{1}-y\right\|+\left(B_{1}-A_{1}\right) / 2,
$$

then $A_{1}+\gamma<\left(A_{1}+B_{1}\right) / 2 \leqq\left\|x_{1}-y\right\|$ and (5) follows. By (5)

$$
\left(d_{1}\left(r_{1}\right)-A_{1}-\gamma\right) r_{2} / r_{1}+A_{1}+\gamma>d_{1}\left(r_{1}\right) \geqq d_{1}\left(r_{2}\right),
$$


thus there exits $y^{\prime} \in M_{1}$ so that

$$
\left(d_{1}\left(r_{1}\right)-A_{1}-\gamma\right) r_{2} / r_{1}+A_{1}+\gamma>\left\|y^{\prime}-x_{2}\right\| \geqq d_{1}\left(r_{2}\right) .
$$

Consider now the function

$$
\psi(s)=\left\|y^{\prime}-(1-s) u_{1}(t)-s u_{2}(t)\right\|, \quad s \in[0,1] .
$$

Since $\psi$ is strictly convex and $0<r_{1}<r_{2}<1$, then

$$
\psi\left(r_{1}\right)<\left(1-\frac{r_{1}}{r_{2}}\right) \psi(0)+\frac{r_{1}}{r_{2}} \psi\left(r_{2}\right) .
$$

As $\psi(0)<\phi_{1}(t, 0)+\gamma$ and (6) holds true, then

$$
\begin{aligned}
\left\|y^{\prime}-x_{1}\right\| & <\left(1-\frac{r_{1}}{r_{2}}\right)\left(A_{1}+\gamma\right)+\frac{r_{1}}{r_{2}}\left(\left(d_{1}\left(r_{1}\right)-A_{1}-\gamma\right) \frac{r_{2}}{r_{1}}+A_{1}+\gamma\right) \\
& =d_{1}\left(r_{1}\right)=d\left(x_{1}, M_{1}\right),
\end{aligned}
$$

a contradiction. Therefore, $d_{1}(s)$ is strictly increasing on the line segment $\left[s_{1}(t), s_{2}(t)\right]$.

Analogously one can show that if $M_{2}$ is an arbitrary closed and nonempty subset of $B\left[y_{2}, \gamma\right]$, then the function $d_{2}(s)=d\left((1-s) u_{1}(t)+s u_{2}(t), M_{2}\right)$ is strictly decreasing on $\left[s_{1}(t), s_{2}(t)\right]$. Thus $d(s)=d_{1}(s)-d_{2}(s)$ increases strictly on $\left[s_{1}(t), s_{2}(t)\right]$.

Furthermore,

$$
d\left(s_{1}(t)\right) d\left(s_{2}(t)\right)<0, \quad t \in \Delta .
$$

In fact, let $t \in \Delta$ and let $M_{i} \subset B\left[y_{i}, \gamma\right], M_{i} \in \mathscr{B}(X), i=1,2$ be arbitrary. Then, by virtue of (3) and (4),

$$
\begin{aligned}
d_{1}\left(s_{1}(t)\right) & =d\left(t y_{1}, M_{1}\right) \leqq\left\|t y_{1}-y_{1}\right\|+\gamma \leqq C_{1}+\gamma<D_{1}-\gamma \\
& \leqq\left\|t y_{1}-y_{2}\right\|-\gamma \leqq d\left(t y_{1}, M_{2}\right)=d_{2}\left(s_{1}(t)\right)
\end{aligned}
$$

i.e. $d\left(s_{1}(t)\right)<0$.

By a similar argument, one shows that $d\left(s_{2}(t)\right)>0$, thus (7) is proved. In view of (7) and the continuity of $d(s)$ on $\left[s_{1}(t), s_{2}(t)\right]$ it follows that there is a unique point $s_{0} \in\left(s_{1}(t), s_{2}(t)\right)$ such that $d_{1}\left(s_{0}\right)=d_{2}\left(s_{0}\right)$. Then for each $t \in \Delta$ there is a unique $x(t) \in\left[t y_{1}, t y_{2}\right] \cap \sigma\left(M_{1}, M_{2}\right)$. The proof is completed.

Lemma 2. Given $y_{1}, y_{2} \in X, \quad\left\|y_{1}\right\|=\left\|y_{2}\right\|, \quad y_{1} \pm y_{2} \neq \theta, \quad$ and $\varepsilon>0$, there is $\gamma \in\left(0,\left\|y_{1}-y_{2}\right\| / 2\right)$ such that $\mathcal{O}\left[\left\{y_{1}, y_{2}\right\}, \gamma\right]$ contains a residual subset $\mathscr{W}$ in the relative Hausdorff topology with the property: For every $M \in \mathscr{W}$ there exists a set $C_{M} \subset B(\theta, \varepsilon)$ of cardinality not smaller than the continuum such that setting $M_{i}=M \cap B\left[y_{i}, \gamma\right], i=1,2$, we have $C_{M} \subset \sigma\left(M_{1}, M_{2}\right)$ and $P\left(\cdot, M_{i}\right)$ are single-valued and upper semi-continuous on $C_{M}$.

Proof. Choose $t_{0} \in(0,1)$ so that $\left[t_{0} y_{1}, t_{0} y_{2}\right] \subset B(\theta, \varepsilon)$ and apply the previous lemma to $y_{1}, y_{2}$ and $t_{0}$. The constants $\gamma$ and $\delta$ are derived from Lemma 1. Without loss of generality we can assume

$$
\begin{aligned}
& Q=\operatorname{co}\left\{\left(t_{0}+k \delta\right) y_{i}: i=1,2 ; k= \pm 1\right\} \subset B(\theta, \varepsilon), \\
& B\left[y_{i}, \gamma\right] \cap B(\theta, \varepsilon)=\oslash, \quad i=1,2 .
\end{aligned}
$$


For $M \in \mathcal{O}\left[\left\{y_{1}, y_{2}\right\}, \gamma\right]$, set $M_{i}=M \cap B\left[y_{i}, \gamma\right], i=1,2$ and observe that $M_{1}, M_{2} \in \mathscr{B}(X)$. Consider the space

$$
\mathscr{S}=\left\{(x, M): x \in Q \cap \sigma\left(M_{1}, M_{2}\right), \quad M \in \mathcal{O}\left[\left\{y_{1}, y_{2}\right\}, \gamma\right]\right\}
$$

with the metric $\rho$ induced by the square metric of $X \times \mathscr{B}(X)$, i.e. $\rho((x, M),(y, N))=$ $\max \{\|x-y\|, H(M, N)\}$. To avoid ambiguity we make use of a subscript letter when denoting balls in $(\mathscr{S}, \rho)$. It is not difficult to check that $\mathscr{S}$ is a complete metric space, and as a simple consequence of Lemma 1 , it is not empty.

Consider also the space $\mathscr{T}=\Delta \times \mathcal{O}\left[\left\{y_{1}, y_{2}\right\}, \gamma\right]$, where $\Delta=\left[t_{0}-\delta, t_{0}+\delta\right]$, and define a mapping $\chi: \mathscr{T} \rightarrow \mathscr{S}$ by $\chi(t, M)=(x(t), M)$ where $x(t) \in\left[t y_{1}, t y_{2}\right] \cap \sigma\left(M_{1}, M_{2}\right)$. According to Lemma $1 \chi$ is well defined and continuous in both directions, thus $\mathscr{S}$ is actually the homeomorphic image of $\mathscr{T}$ via $\chi$.

Let for $n \in \mathbb{N}$

$$
\begin{aligned}
& \mathscr{U}_{n}=\{(x, M) \in \mathscr{S}: \text { there is } r>0 \text { such that } \\
& \left.\operatorname{diam}\left(B\left[x, d\left(x, M_{i}\right)+r\right] \cap M_{i}\right)<n^{-1}, \quad i=1,2\right\} .
\end{aligned}
$$

We claim that $\mathscr{U}_{n}$ contains an open and dense subset of $\mathscr{S}$. To prove it fix $n \in \mathbb{N}, \kappa>0$ and $\left(x_{0}, M^{0}\right) \in \mathscr{S}$, i.e. $x_{0} \in \sigma\left(M_{1}^{0}, M_{2}^{0}\right)$ where $M_{i}^{0}=M^{0} \cap B\left[y_{i}, \gamma\right]$ for $i=1,2$.

For the claim, it suffices to show that $B_{\mathscr{S}}\left(\left(x_{0}, M^{0}\right), \kappa\right) \cap \mathscr{U}_{n}$ contains an open ball in $\mathscr{S}$. Since $\chi$ is continuous there are $c \in(0,1)$ and $x_{1} \in Q$ such that setting $M_{i}^{\prime}=y_{i}+c\left(M_{i}^{0}-y_{i}\right)$, $i=1,2$ and $M^{\prime}=M_{1}^{\prime} \cup M_{2}^{\prime}$ we have

$$
\begin{aligned}
& \left(x_{1}, M^{\prime}\right) \in \mathscr{S}, \quad x_{1} \in \sigma\left(M_{1}^{\prime}, M_{2}^{\prime}\right), \\
& \left\|x_{0}-x_{1}\right\|<\kappa / 2, \\
& 0<\lambda<\min \{3 \kappa / 10,6 / n\}, H\left(M_{i}^{0}, M_{i}^{\prime}\right) \leqq \lambda,
\end{aligned}
$$

and $M_{i}^{\prime} \subset B\left[y_{i}, \gamma-\lambda\right], i=1,2$. Take

$$
z_{i} \in M_{i}^{\prime} \cap B\left(x_{1}, d+\lambda / 3\right), \quad i=1,2,
$$

where $d=d\left(x_{1}, M_{i}^{\prime}\right)$, and put

$$
\left\{v_{i}\right\}=\left[z_{i}, x_{1}\right] \cap S\left(x_{1}, d-\lambda / 3\right) \text { for } i=1,2 .
$$

It is easy to see that

$$
\left\|v_{i}-z_{i}\right\|<2 \lambda / 3, \quad i=1,2 .
$$

Since $z_{i} \in B\left[y_{i}, \gamma-\lambda\right]$, then $v_{i} \in B\left(y_{i}, \gamma-\lambda / 3\right), i=1,2$. Let

$$
N=\left\{v_{1}\right\} \cup M_{1}^{\prime} \cup\left\{v_{2}\right\} \cup M_{2}^{\prime} \text {. }
$$

Obviously $\left(x_{1}, N\right) \in \mathscr{S}$. Since $H\left(M_{i}^{0}, M_{i}^{\prime}\right) \leqq \lambda$, and by (9) $d\left(v_{i}, M_{i}^{\prime}\right)<2 \lambda / 3$, then $H\left(M^{0}, N\right)<5 \lambda / 3$ and

$$
\rho\left(\left(x_{0}, M^{0}\right),\left(x_{1}, N\right)\right)<\max \{\kappa / 2,5 \lambda / 3\}=\kappa / 2,
$$

according to (8). Consequently, $B_{\mathscr{S}}\left(\left(x_{1}, N\right), \lambda / 12\right) \subset B_{\mathscr{S}}\left(\left(x_{0}, M^{0}\right), \kappa\right)$.

We intend to prove that $B_{\mathscr{S}}\left(\left(x_{1}, N\right), \lambda / 12\right)$ is contained in $\mathscr{U}_{n}$. Take $(x, M) \in B_{\mathscr{S}}\left(\left(x_{1}, N\right), \lambda / 12\right)$ and let $M_{i}=M \cap B\left[y_{i}, \gamma\right], i=1,2$. Then $\left\|x-x_{1}\right\|<\lambda / 12$ and $x \in \sigma\left(M_{1}, M_{2}\right)$. 
For $i=1,2$ let

$$
V_{i}=M_{i} \cap B\left[v_{i}, \lambda / 12\right], \quad W_{i}=M_{i} \cap\left(\cup_{y \in M_{i}^{\prime}} B(y, \lambda / 12)\right) .
$$

Since by definition $d\left(v_{i}, M_{i}^{\prime}\right) \geqq \lambda / 3$, then $M_{i}=V_{i} \cup W_{i}, V_{i} \cap W_{i}=\oslash$ and $V_{i}, W_{i}$ are closed and nonempty subsets of $B\left[y_{i}, \gamma\right]$ for $i=1,2$.

Further,

$$
\begin{aligned}
& d=d\left(x_{1}, M_{i}^{\prime}\right) \leqq\left\|x_{1}-x\right\|+d\left(x, W_{i}\right)+H\left(W_{i}, M_{i}^{\prime}\right)<\lambda / 6+d\left(x, W_{i}\right), \text { i.e. } \\
& d\left(x, W_{i}\right)>d-\lambda / 6, \quad i=1,2 .
\end{aligned}
$$

On the other hand, for each $z \in V_{i}$ (where $i$ is fixed) we have $\|x-z\| \leqq\left\|x-x_{1}\right\|+$ $\left\|x_{1}-v_{i}\right\|+\left\|v_{i}-z\right\|<d-\lambda / 6$, whence

$$
V_{i} \subset B[x, d-\lambda / 6], \quad i=1,2 \text {. }
$$

Let $\quad r$ satisfy $d-\lambda / 6=d\left(x, V_{i}\right)+r$. It is easy to check that $r>0$. Since $\operatorname{diam} V_{i} \leqq \lambda / 6<n^{-1}$, taking into account (10) and (11), we conclude that $d\left(x, M_{i}\right)=d\left(x, V_{i}\right)$ and

$$
\operatorname{diam}\left(B\left[x, d\left(x, M_{i}\right)+r\right] \cap M_{i}\right)<1 / n \text { for } i=1,2,
$$

i.e. $(x, M) \in \mathscr{U}_{n}$. The claim is proved.

Finally, put $\mathscr{U}=\bigcap_{n=1}^{\infty} \mathscr{U}_{n}$. $\mathscr{U}$ is a residual subset of $\mathscr{S}$ and if $(x, M) \in \mathscr{U}$ then for $M_{i}=M \cap B\left[y_{i}, \gamma\right]$ the projections $P\left(\cdot, M_{i}\right)$ are continuous at $x$ (i.e. single-valued and upper semi-continuous at $x$ ), $i=1,2$.

Now denote by $\mathscr{V}$ the residual subset of $\mathscr{T}$ corresponding to $\mathscr{U}$ via $\chi^{-1}$. By KuratowskiUlam theorem [8] there exists a residual subset $\mathscr{W}$ of $\mathcal{O}\left[\left\{y_{1}, y_{2}\right\}, \gamma\right]$ such that for each $M \in \mathscr{W}$ there is a residual subset $T_{M}$ of $\Delta$ with the property that $(t, M) \in \mathscr{V}$ whenever $t \in T_{M}$. The set $D_{M}=\left\{(t, M): t \in T_{M}\right\}$ contains at least a continuum of elements. Denote by $C_{M}$ the set of the first coordinates of the image set $\chi\left(D_{M}\right)$. Since for $M \in \mathscr{W}$ and $t \in T_{M}$ $\chi(t, M)=(x(t), M) \in \mathscr{U}$ and $x(t) \in Q \subset B(\theta, \varepsilon)$, we conclude that for any $M \in \mathscr{W}$ the set $C_{M} \subset B(\theta, \varepsilon)$ is of cardinality not smaller than the continuum and for $M_{i}=M \cap B\left[y_{i}, \gamma\right]$, $i=1,2$, the metric projections $P\left(\cdot, M_{i}\right)$ are continuous at $x \in C_{M} \subset \sigma\left(M_{1}, M_{2}\right)$. This completes the proof.

Lemma 3. Given $M_{0} \in \mathscr{B}(X), a \in X, \eta>0$ and $\varepsilon>0$, there are $M_{1} \in \mathscr{B}(X)$ and $\kappa>0$ such that:

$$
\mathcal{O}\left[M_{1}, \kappa\right] \subset \mathcal{O}\left(M_{0}, \eta\right)
$$

and there exists a residual subset $\mathscr{R}(a, \varepsilon)$ of $\mathcal{O}\left[M_{1}, \kappa\right]$, with respect to the relative Hausdorff topology, such that for every $M \in \mathscr{R}(a, \varepsilon)$ the two-locus $L^{2} M$ contains a continuum of points in $B(a, \varepsilon)$ at which $P(\cdot, M)$ is upper semi-continuous.

Proof. With no loss of generality, suppose $B(a, \eta) \cap M_{0}=\oslash$. Otherwise use the argument below with $M_{0}^{\prime}=M_{0} \backslash B(a, \eta / 2)$ instead of $M_{0}$ and $\eta^{\prime}=\eta / 2$ instead of $\eta$, provided $M_{0}^{\prime} \neq \oslash$. The case $M_{0}^{\prime}=\oslash$ is easily reduced to Lemma 2 .

For the sake of brevity, put $d=d\left(a, M_{0}\right)$. Take $z \in M_{0}$ such that $\|a-z\|<d+\eta / 6$ and $v \in X$ such that $\{v\}=[a, z] \cap S(a, d-\eta / 3)$. It follows that

$$
d\left(v, M_{0}\right) \leqq\|v-z\|<\eta / 2 \text {. }
$$


As $\operatorname{dim} X \geqq 2$, the last inequality guarantees the existence of two points $v_{i} \in S(a, d-\eta / 3)$ satisfying

$$
d\left(v_{i}, M_{0}\right)<\eta / 2, \quad i=1,2
$$

and $v_{1}-a \neq \pm v_{2}-a$. Moreover,

$$
d\left(v_{i}, M_{0}\right) \geqq \eta / 3, \quad i=1,2 .
$$

Apply Lemma 2 with $y_{i}=v_{i}-a, i=1,2$, and $\varepsilon_{0}=\min \{\varepsilon, \eta / 12\}$. There are $\gamma>0$ and a residual subset $\mathscr{W}$ of $\mathcal{O}\left[\left\{v_{1}, v_{2}\right\}, \gamma\right]$, with respect to the relative Hausdorff topology, such that $M \in \mathscr{W}$ implies $\operatorname{card}\left(L^{2} M \cap \operatorname{usc} M \cap B\left(a, \varepsilon_{0}\right)\right) \geqq \mathbf{c}$.

Set $\kappa=\min \{\gamma, \eta / 12\}$. Then $\mathscr{W}^{\prime}=\mathscr{W} \cap \mathcal{O}\left[\left\{v_{1}, v_{2}\right\}, \kappa\right]$ is also residual in the smaller ball.

Let $M_{1}=M_{0} \cup\left\{v_{1}, v_{2}\right\}$. It follows from (13) and the choice of $\kappa$ that (12) holds true. Define now

Since

$$
\mathscr{R}(a, \varepsilon)=\left\{M \in \mathcal{O}\left[M_{1}, \kappa\right]: M \cap\left(\cup_{i=1,2} B\left[v_{i}, \kappa\right]\right) \in \mathscr{W}^{\prime}\right\} .
$$

$$
\operatorname{cl}\left(\cup\left\{B(x, \kappa): x \in M_{0}\right\}\right) \cap\left(\cup_{i=1,2} B\left[v_{i}, \kappa\right]\right)=\oslash,
$$

then $\mathscr{R}(a, \varepsilon)$ is residual in $\mathcal{O}\left[M_{1}, \kappa\right]$ with respect to the relative Hausdorff topology. In order to show that $\mathscr{R}(a, \varepsilon)$ satisfies the statement of the lemma, we only need to prove

$$
P(x, M) \subset \bigcup_{i=1,2} B\left[v_{i}, \kappa\right], \text { whenever } x \in B\left(a, \varepsilon_{0}\right), \quad M \in \mathcal{O}\left[M_{1}, \kappa\right] .
$$

But (15) follows from (14) and the choice of $\kappa$, since for $x \in B\left(a, \varepsilon_{0}\right)$

and

$$
d\left(x, M \cap B\left[v_{i}, \kappa\right]\right)<d-\eta / 6, \quad i=1,2
$$

$$
d\left(x, M \backslash \cup_{i=1,2} B\left[v_{i}, \kappa\right]\right)>d-\eta / 6 .
$$

The proof is complete.

The following lemma states roughly speaking, that a set is "globally residual" if it is "locally residual". This is true under more general assumptions [6 (V. I, 2, 10)]. In our setting, the proof is short and for the sake of completeness is included.

Lemma 4. Suppose $Z$ is a complete metric space and $R \subset Z$ satisfies the property: For every $x \in Z$ and every $\eta>0$ there are $y \in Z$ and $\kappa>0$ such that $B[y, \kappa] \subset B(x, \eta)$ and $R \cap B[y, \kappa]$ is residual in $B[y, \kappa]$ with respect to the relative topology. Then $R$ is a residual subset of $Z$.

Proof. Let $\mathscr{F}$ be the family of all nonempty closed balls in $Z$ such that $B \in \mathscr{F}$ implies $R \cap B$ is residual in $B$ with respect to the relative topology. Consider the partially ordered (by inclusion) set $\mathscr{G}$ whose elements are sets of pairwise disjoint balls from $\mathscr{F}$, i.e. for $g \in \mathscr{G}$ there is a nonempty index set $I$ such that

$$
g=\left\{B_{i} \in \mathscr{F}: j \neq k \text { implies } B_{j} \cap B_{k}=\oslash, i, j, k \in I\right\} .
$$

Make use of Zorn lemma to ensure the existence of a maximal element $h$ of $\mathscr{G}$. The set $\cup_{B \in h} B$ contains a dense $G_{\delta}$ subset of $Z$ which is a subset of $R$, so $R$ is residual in $Z$.

4. Proof of theorem. Let $X$ be a separable strictly convex Banach space with $\operatorname{dim} X \geqq 2$. 
By Lemma 3 and Lemma 4 for every $a \in X$ and every $\varepsilon>0$ there is a residual subset $\mathscr{R}(a, \varepsilon)$ of $\mathscr{B}(X)$ such that

$$
\operatorname{card}\left(L^{2} M \cap \operatorname{usc} M \cap B(a, \varepsilon)\right) \geqq \mathbf{c}
$$

whenever $M \in \mathscr{R}(a, \varepsilon)$.

Let $\left\{a_{1}, a_{2}, \ldots\right\}$ be a countable dense subset of $X$. Put

$$
\mathscr{R}=\bigcap_{n=1}^{\infty} \mathscr{R}\left(a_{n}, n^{-1}\right) .
$$

For every $M \in \mathscr{R}$ the two-locus $L^{2} M$ intersects an arbitrary nonempty open subset $U$ of $X$ at a set containing at least a continuum of points of upper semi-continuity of $P(\cdot, M)$, i.e. the set $C_{M}=L^{2} M \cap$ usc $M$ is everywhere continual. The theorem is proved.

\section{References}

[1] F. S. DE Blasi and J. MYJAK, Ambiguous loci of the nearest point mapping in Banach spaces. Arch. Math. 61, 377-384 (1993).

[2] F. S. DE Blasi, P. S. Kenderov and J. MyjaK, Ambiguous loci of the metric projection on compact starshaped sets in a Banach space. Monatsh. Math. 119, $23-36$ (1995).

[3] J. M. BorweIn, Weak local supportability and applications to approximation. Pacific J. Math. 82, $323-338$ (1979).

[4] J. M. Borwein and S. FitzPATRick, Existence of nearest points in Banach spaces. Canad. J. Math. 41, $702-720$ (1989).

[5] S. V. Konyagin, On approximation of closed sets in Banach spaces and the characterization of strongly convex spaces. Soviet Math. Dokl. 21, 418-422 (1980).

[6] K. Kuratowski, Topology. Vol. I. New York-London 1966.

[7] Ka-Sing LaU, Almost Chebyshev subsets in reflexive Banach spaces. Indiana Univ. Math. J. 27, $791-795$ (1978).

[8] J. C. Охтовy, Measure and Category. Berlin 1971.

[9] S. B. Stechkin, Approximative properties of subsets of Banach spaces. Rev. Roum. Pures Appl. 8, 5-8 (1963) (in Russian).

[10] T. ZAMFIRESCU, The nearest point mapping is single-valued nearly everewhere. Arch. Math. 54, $563-566$ (1990).

[11] N. V. Zhivkov, Compacta with dense ambiguous loci of metric projections and antiprojections. Proc. Amer. Math. Soc. 123, 3403-3411 (1995).

[12] N. V. Zhivkov, Densely two-valued metric projections in uniformly convex Banach spaces. SetValued Anal. 3, 195-209 (1995).

Eingegangen am 12. 11. 1997

Anschriften der Autoren:

F. S. de Blasi

Dipartimento di Matematica

Università di Roma II

Via della Ricerca Scientifica

I-00133 Roma

Italy
N. V. Zhivkov

Institute of Mathematics

Bulgarian Academy of Sciences

Sofia 1113

Bulgaria 